\title{
The Effectiveness of Augmented Reality Technology (AR) in Reducing Attention Deficiency Disorder (ADD) for Children with Learning disabilities (LD)
}

\section{Eman M.E. Ahmed (PhD.)}

Casework Department

Higher Institute of Social Work-Alexandria 


\section{The Effectiveness of Augmented Reality Technology (AR) in} Reducing Attention Deficiency Disorder (ADD) for Children with

\section{Learning disabilities (LD)}

\section{Eman M.E. Ahmed (PhD.)}

Casework Department

Higher Institute of Social Work-Alexandria

\section{Abstract:}

The present study aims at determining the effectiveness of Augmented Reality (AR) technique in reducing Attention Deficiency Disorder among children with learning difficulties. The study sample consisted of 20 children with learning difficulties they were distributed among two groups, control and experiment, each consisting of 10 children, aging between 6 and 9 years. The children were provided with computers for learning and activities, as well as, 3D glasses for watching educational and behavioral movies. The current study used the following tools: Professional interview and Attention deficiency scale. The study concluded that the children achieved changes in levels of concentration and improvement in their follow up of their lessons, as well as, their participation in different activities and tasks. The results also showed the impact of AR on the attention deficiency, more than on the impulsive behavior and the hyperactivity.

Keywords: Augmented Reality (AR), Attention Deficit Disorder (ADD). Learning disabilities (LD).

\section{Introduction}

Specific learning disabilities have been recognized in some countries for much of the 20th century, in other countries only in the latter half of the century, and yet not at all in other places. Even where they have been recognized, the amount of help available varies from no services to their universal provision. (Crealock\& Kronick1993, P.5)

For more than 35 years, the National Center for Learning disabilities (NCLD) has provided essential information and needed resources and services to the learning disabilities community. And in response to a rapidly changing educational landscape, it has recently broadened its mission to address the needs of the more than one in five children, adolescents and adults who are impacted by learning and attention issues every day, in school, at home, in the community and in the workplace (Alper, Lea, Schloss, 2000, P.3). 
The most common types of specific learning disabilities are those that impact the areas of reading, math and written expression. They may co-occur with other disorders of attention, language and behavior, but are distinct in how they impact learning. Common characteristics include: (Pierangelo \& Giulian 2007, P.8): Dyslexia, Dyscalculia, Auditory Processing Deficit or Disorder, Visual Processing Deficit or Disorder, Non-Verbal Learning disabilities is, Executive Functioning Deficits, Attention Deficit/Hyperactivity Disorder (ADHD) that results in significant inattention, hyperactivity, distractibility or a combination of these characteristics. It is estimated that as many as one-third of those with LD also have ADHD.

There are many studies have tackled the issue of developing the skills of children with learning disabilities such as social skills and academic achievement (Hall 2015), the concept of selfdevelopment(Gorges, Neumann, Wild, Stranghöner, Lütje, 2018), and training the working memory (Chen, Ye, Chang, Chen, Zhou, 2018).

Martínez-Álvarez,(2017) also referred to improving learning among bilingual children with learning disabilities. Also (Tulu \&Ergui 2015) confirmed the effects of non- verbal signals on children with learning disabilities and a study by McKeever, 2017 also mentioned the influence of classical music on children with learning disabilities.

Many studies discussed the needs of parents of children with learning disabilities such as (Auriemma2016, Seema 2017 and Valle 2018).

Some students with learning disabilities have disabilities focusing, sustaining and shifting attention. These disabilities might result from physical discomfort, emotional issues, interest and motivational factors or from challenges with self-regulation. Persistent patterns of disabilities such as inattention, hyperactivity, or impulsiveness, or any combination of these, might be the result of a biologically based condition known as Attention Deficit Hyperactivity Disorder. ADHD is a regulatory problem of attention, activity level and impulse control, and it can have a significant impact on a student's ability to learn. (Butler, Miller, Crehan, Babbitt, Pierce, 2003, P.99)

The study of Friedman et al. 2018 also confirmed the significance working memory for both reading and resolving mathematical problems among ADHD children. (Friedman, Rapport, Calub, Eckrich, 2018, P. 65) 
According to recent data from the Centers for Disease Control and Prevention: About 6.4 million children have received an ADHD diagnosis at some point. ADHD diagnoses have increased 16 percent since 2007 and 53 percent in the past decade. Boys (13.2 percent) were more likely than girls (5.6 percent) to have ever been diagnosed with ADHD. Rates of ADHD diagnosis increased at a greater rate among older teens as compared to younger children.( Horowitz 2014, P.5)

The causes for attention deficit disorders are not fully understood yet, but heredity is known to be a key factor (Tripp \& Wickens, 2009). Apparently, the child's hereditary tendencies, together with the environment and its interactional relationships, affect the way and extent to which the child's disabilities manifest themselves in daily life. (Kemp, Smith, Segal, 2015, P.65)

Problems in attention and behavior regulation appear when the demands of the environment increase, as is often the case at school. To understand the disabilities and define their causes, it is good for the assessor to know about the child's earlier development as well. Even according to the definition of ADHD, the child must have had signs of the difficulty before school-age. (Aro \& Ahonen 2011, P.86).

Mason \& Joshi (2018) emphasized the importance of selecting the right methods to help children with ADHD.

One of the most important aspects of technology in education is its ability to level the field of opportunity for students Technology can be a powerful tool for transforming learning. It can help affirm and advance relationships between educators and students, reinvent our approaches to learning and collaboration, shrink long-standing equity and accessibility gaps, and adapt learning experiences to meet the needs of all learners(King, Joseph, Katrina, 2017, P.11).Technology is not a strategy in and of itself; rather, it is an adaptable and powerful tool for providing appropriate personalized learning activities and adaptations, thus helping them learn new vocabulary, make connections with new knowledge and maintain enjoyment, curiosity. (Ribble \& Bailey 2015.P.13)

Augmented reality is (AR) the technology of the future which could have a significant impact on the education. By using the technology it is possible visually reproduce processes or objects that are difficult or almost impossible to recreate in the real world and make the learning experience more fascinating and understandable. Moreover, it could affect students motivation and learning outcome by applying five main components of the progressive learning process (high-quality 
content, object visualization, gamification and game-based learning, realtime performance and interaction). (Roksolana2017, P.3)

Augmented Reality (AR) has been defined as a variation of VR (Azuma, 1997.p25). While VR completely immerses the user inside a computer-generated environment where they cannot relate to the physical environment, AR allows the overlaying of virtual elements onto the physical environment. Azuma (1997) stated that the most common characteristics of AR are that it combines real and virtual elements, it is interactive in real time, and it registers real and virtual objects with each other. (Azuma 1997, P. 26)

A number of studies have explored desktop AR solutions for a variety of uses including education, medicine, and design education (Burke et al. 2010; Chandrasekera \&Yoon 2014; Juanesetal.2014), few attempts have been made to explore the possibilities of using AR in architecture and interior design education.

A desktop AR system uses a webcam, a desktop or laptop computer, and afiducial marker. The virtual object is overlaid on the marker and displayed on the computer screen. In most cases the webcam is facing the viewer. The cost-effective nature of desktop AR has provided the opportunity for its use in many domains. The number of open source and free or cost-effective software solutions that allow creating these desktop AR experiences have also improved the accessibility of this technology. Previous studies have shown that not only can these type of AR experiences be used in design education but such experiences have been shown to be preferred by design students as an instructional medium as and to improve their spatio-cognitive abilities (Chandrasekera\& Yoon 2015; Martín et al. 2010).

According to Yonezawa \&Tokuda (2013), it is important to show the smartness of ubiquitous computing environment to people who don't know about the environment. To effectively provide this information to the users, we propose an interface called Secondsighted Glasses. The glasses show users the future effects of each action they may take with augmented reality technique.

Also the studies of Olga S., Julie M., Cameron B. (2017), Al-Asheeri (2017), mentioned the Effectiveness of Using Augmented Reality in Teaching the English to Children. As per the study of Qian (2018), the results showed that visual communication based on augmented reality could provide a new form of teaching demonstration and enrich the content of classroom teaching. To sum up, this method improves the students' participation and enthusiasm, and enhances the teaching effect. 
Major focus of AR applications for children is enhancing learning activities, as early childhood activities center around cognitive and socioemotional development (Wood \& Attfield 2005). There are several AR advantages to enhance these learning activities. First, AR enables children to get access to knowledge without switching attention between virtual and physical contexts. This accelerates the interaction and feedback processes in learning (Roschelle, Pea, Hoadley, Gordin, Means, 2000) for children who have a smaller working memory and weaker attentional control than adults (Whitebread 2012). Second, AR supports learning knowledge in three-dimensional space, which is beneficial for young children whose spatial cognition is still under development (Cohen 2013). Third, the tangible feature of AR enables children to directly experiment with knowledge components, which is considered essential for conceptualization development (Glenberg, Gutierrez, Levin, Japuntich, Kaschak, 2004), and provides playful learning experiences as it is more accessible to young children than traditional keyboard and mouse interfaces (Marshall 2007) and the study of Boyce (2016) also mentioned the impact of a video game called Quizlet on acquiring scientific vocabulary among children with learning disabilities. Also the study of Elsaigh \& Elsiddig (2017) mentioned the role of e- learning in improving the reading skills among children with learning disabilities. Bikic, Leckman, Christensen, Bilenberg, Dalsgaard (2018) also referred to the positive effects of cognitive training using computer with children with ADHD.

According to The study of Yilmaz, Kucuk, Goktas (2017) augmented reality picture books (ARPB), are attractive and evocative for children, who perceive them as magic and more enjoyable than conventional books. This study demonstrates how they can be used as effective educational tools to improve preschool children's cognition and listening skills.

The present study is different from previous ones because it aimed at: 1-Identifying the effectiveness of the Augmented Reality technique in reducing the Attention deficiency among children with learning disabilities. 2-Recommendingmethods of improving Augmented Reality in teaching children with children with learning disabilities.

The significance of the current study shows through: 1. Examining a coexisting issue, that is, the effectiveness of Augmented Reality (AR) in education and its positive impact counteracting the negative aspects of Virtual Reality (VR), 2. It is aimed at a certain sensitive educational stage, Primary Education, 
during which the student forms basic characteristics which are important and must be considered during educating them, 3. The results referred to by the statistics which showed the increase of children with learning disabilities and 4 . The increase in attention deficiency problems among children with learning disabilities and the fact that it is influenced by impulsiveness and hyperactivity.

\section{Study Hypotheses:}

First Hypothesis: There are differences between the mean values of experimental group and the mean values of the control group according to the attention disorder scale and its sub- scales; attention deficiency, impulsions, hyperactivity, favoring the experimental group.

Second Hypothesis: There are differences between the mean values of the experimental group on the pre and post measurements on the attention disorder scale and it sub- scales attention deficiency, impulsion, hyperactivity.

Third Hypothesis: There are no differences between the mean values of the control group pre and post measurements on the attention disorder scale and its sub- scales attention deficiency, impulsions, hyperactivity.

\section{Study Concepts:}

1- Learning disabilities (LD): According to the Learning disabilities Association of Canada (LDAC), Learning disabilities refer to a number of disorders, which may affect the acquisition, organization, retention, understanding, or use of verbal or nonverbal information including: oral language; reading; written language; mathematics; social skills; organizational abilities. (Fiedorowicz et al. 2015,P.15).

California community colleges define learning disability as "a persistent condition of presumed neurological dysfunction, which may also exist with other disabling conditions. This dysfunction continues despite instruction in standard classroom situations." (Hayes 1993,P.5).

According to the current study Learning Disabilities (LD) is defined as a disorder in one or more of the basic psychological processes participating in understanding, using, speaking or writing a language, which are manifested in Attention Deficiency Disorder among those children.

2- Attention Deficit Disorder (ADD): This disability defined by the American Psychiatric Association in its Diagnostic and Statistical Manual of Mental Disorders which is commonly referred to as the DSM-IV, as: A. "Either (1) or (2): (Daly et al. 2007, P. 73-89.) 
(1) Six or more of the following symptoms of inattention have persisted for at least 6 months to a degree that is maladaptive and inconsistent with developmental level: Inattention: give close attention to details or makes careless mistakes in schoolwork, difficulty sustaining attention in tasks, does not seem to listen when spoken to directly, does not follow through on instructions and fails to finish schoolwork, has difficulty organizing tasks, avoids, dislikes, or is reluctant to engage in tasks that require sustained mental effort, loses things necessary for tasks, easily distracted by extraneous stimuli, forgetful in daily activities.

(2) Six or more of the following symptoms of hyperactivityimpulsiveness have persisted for at least 6 months to a degree that is maladaptive and inconsistent with developmental level: Hyperactivity: fidgets with hands or feet or squirms in seat, leaves seat in classroom, runs about or climbs excessively in situations in which it is inappropriate, has difficulty playing or engaging in leisure activities quietly," on the go" or acts as if "driven by a motor. Impulsiveness: blurts out answers before questions have been completed, has difficulty awaiting turn, interrupts or intrudes on others. Some hyperactive-impulsive or inattentive symptoms that caused impairment were present beforeage 7years. Some impairment from the symptoms is present in two or more settings. There must be clear evidence of clinically significant impairment in social, academic, or occupational functioning. The symptoms do not occur exclusively during the course of a Pervasive Development Disorder, Schizophrenia, or other Psychotic Disorder and are not better accounted for by another mental disorder.

Attention disorder definition according to this study: determined through:

- Attention deficiency: that is the difficulty in focusing attention of the activity performed by the child and being easily influenced by external factors.

- Impulsion: child's fast response without thinking, not waiting for his/ her turn, interrupting others during playing or talking.

- Hyperactivity: child's inability to settle down in one place or to sit quietly and excessive movement. 
3- Augmented Reality (AR): Azuma (1997) defined AR as a variation of VR technology that supplements reality by superimposing virtual objects into it. $A R$ is an interface that allows tangible interaction through the use of fiducial markers. This is also termed "desktop augmented reality" in the literature.(Azuma1997, P.355)

Etmeezi (2010) defined AR as computer technology based on the integration of images, scenes, and clips of the real world with the virtual world through three-dimensional computer graphics, where the computer controls these components. (Etmeezi 2010, P.55)

Yuen, Yaoyuneyong, Johnson (2011) identified AR as a form of technology that enhances the real world through content produced by the computer. AR technology enables the seamless addition of digital content to the user's perception of the real world. Two-dimensional and threedimensional shapes can be added in addition to audio, video, animation, and/or text. These enhancements work to expand individual knowledge and understanding of what is happening around them. (Yuen, Yaoyuneyong, Johnson 2011, P.119).

This study defines AR as a computer technology that depend upon merging images and scenes from real world into virtual world through computer $3 \mathrm{D}$ drawings, as the children receive learning and activity through computer.

\section{Theories guiding the study:}

The basic theories underlying the Augmented Reality Technique application in education, Nidal (2012), are;

Behavioral theory (Skinner): according to this theory; a behavior is either learned or modified through learning; so the behavioral theory is concerned with establishing the educational setting and providing the learners with stimulators that motivate them to respond; thus reinforcing such response. The Augmented Reality technology aims at establishing such educational settings through the multimedia which act as learning stimulators.

The constructive theory: the constructive learning settings are strongly related to E- learning in general and to the Augmented Reality in particular. The subject presentation through multimedia enables the construction of concepts through personal activities and observation within the reach interactive settings; thus leading to better learning. So the constructive theory principles include that the learner builds knowledge using the activity he/ she performs through achieving understanding. 
Connectivism Theory: the behavioral, constructive and knowledge theories focus on the learning process taking place inside the learner and they do not take the role of the surrounding environment in teaching and learning into consideration. As a teaching technique that focuses on the how and not the amount of learning emerges, a connectivism theory emerges. It was developed by George Simens and Downe in 2004. The basic principle thereof is the learner's ability to classify and sort knowledgeinto important parts. It considers the networks built as node at least two or more nodes. Each node represents a source for knowledge and they interconnected through links. So learning takes place through learner's ability to reach such links effectively. The Augmented reality technique reliesuponone of the connectivism theory principles, that is, learning can be through non- human tools and devices, as through such smart and portabledevices and there applications learning can take place.(Anderson\& Liarokapis, 2014, P.89)

\section{Intervention program:}

\section{Intervention objectives:}

1. Reducing the attention deficiency disorder among children with learning disabilities, that is defined through the following aspects: attention deficiency, impulsion, and hyperactivity.

2. Linking knowing the activity to understanding it and providing the children with learning environments that use AR technologies in education through deploying computers in education and activity and 3D googles in watching educational and behavioral movies.

\section{Intervention steps:}

1. Providing audio and visual aids for the children through computers and 3D googles for learning and practicing activities.

2. Making arrangements with teachers and social workers to provide extra- curricular activities, during the study period that is over 6 months, using computers and $3 \mathrm{D}$ googles.

Observing the attention deficiency behavior among children and its different aspects; attention deficiency, impulsion and hyperactivity, both before and after the intervention.

\section{Intervention methods:}

According to the theories applies in the present study the following methods were deployed:

1. Positive support: through encouraging the child and giving him/ her a golden star then the child shows attention. 
2. Procedural reduction method: through preventing the support upon the occurrence of attention deficiency behavior, thus leading into gradual reduction of such behavior.

3. Differential support method: providing the child with the positive support upon the improvement of the attention deficiency behaviors.

4. Offering the child, a role model on the behavioral aspect using audio and visual shows through animated movies and using 3D googles to watch them which facilitates understanding and comprehending them and the spontaneous behavior.

5. Deploying continuous advice, instructions and directions to modify children's attention deficiency behaviors.

\section{Assessing the intervention results:}

Through measuring the attention deficiency disorder with its three aspects; attention deficiency, impulsion and hyperactivity among children and comparing post measurements with pre measurements

\section{Methodology:}

This is a Semi-empirical study based on two groups, a control and experiment, chosen by the researcher because of their relationship to the subject of the study. The study population consisted of 40 children with learning difficulties from the Center for Behavioral Adjustment and Learning Disabilities, Alexandria, 20 children were selected through an objective deliberate sample to serve the study objectives and according to the specified sampling conditions with ages ranging 6 to 9 years. Have the highest degree of attention deficit and distributed to two groups. A control group and a trial group, each consisting of 10 children. It was also agreed with the sample's children families to apply the program. This center was chosen because of the presence of the sample and because, during the study period, extending for 6 months, it provided the children with computers for learning and activities, as well as, 3D glasses for watching educational and behavioral movies. The current study used the following tools:

1. Professional interview individual interviews with the experimental group and interviews with teachers and social workers. 2. Attention deficiency scale (prepared by the researcher) the researcher determined the basic dimensions of the scale according to previous scales. According to the research concepts these dimensions were: the first dimension; that is, attention deficiency represented by items 1- 15, the second dimension; impulsiveness, represented by the 
items 16- 27 and third dimension; hyperactivity and represented by items form 28-37. Then the items related to each dimension were determined. The researcher ensured that the scale's items were gradual as each dimension had three responses; "always" (3 points) with highest score, "sometimes" (2 points) with medium score and finally "rarely" (1 point) with the least score. The scale included 37 items distributed among the three dimensions. The highest score for the first dimension was 450, the medium score was 300 and the lowest score thereof was 150. As for the second dimension the highest score was 360 , medium score was 240 and the lowest score was 120 . The highest score for the third dimension was 300 and medium score thereof was 200 and the lowest score was 100 .

Scale validity: the researcher presented the scale to 10 specialized professors in social work to evaluate the items in terms of their wording and relation to the study topic. And accordingly the researcher rephrased some items and canceled some other ones based upon there degree of compliance (not less than $80 \%$ ).

Internal validity $=$ square root of the measurement stability coefficient $\sqrt{ } 0.93=0.97$ Thus referring that the measurement has a high validity degree.

Scale reliability: The researcher employed the test- retest method with 10 teachers of children with learning disabilities in Muharam Bek Behavior modification center, and then retested it again 15 days later. The reliability coefficient was 0.96 . According to correlation significance tables, the degree of freedom was 13 , probability $0.05, \mathrm{R}_{\mathrm{tab}}=0.514$, therefore the correlation was statistically significant.

Table (1): The researcher undertook homogeneity between the control and experimental groups according to the measuring axes and the results were as follows:

\begin{tabular}{||l||c||c||c||c||c||c||}
\hline \hline \multirow{2}{*}{ The measuring axes } & \multicolumn{2}{|c|}{$\begin{array}{c}\text { Experiment } \\
(\mathbf{n}=\mathbf{1 0})\end{array}$} & \multicolumn{2}{c|}{$\begin{array}{c}\text { Control } \\
(\mathbf{n}=\mathbf{1 0})\end{array}$} & \multirow{2}{*}{ t } & \multirow{2}{*}{ P } \\
\cline { 2 - 5 } & Mean & \pm SD & Mean & \pm SD & & \\
\hline \hline Attention & 40.80 & 0.92 & 40.40 & 0.70 & 1.095 & 0.288 \\
\hline \hline Impulsiveness & 31.80 & 0.92 & 31.50 & 1.27 & 0.605 & 0.552 \\
\hline \hline Hyper activity & 26.30 & 0.82 & 25.90 & 0.99 & 0.980 & 0.340 \\
\hline \hline Overall & 98.90 & 1.45 & 97.80 & 1.32 & 1.777 & 0.093 \\
\hline
\end{tabular}

t: Student t-tes p: $\mathrm{p}$ value for comparing between the studied groups 


\section{Results:}

Table (2): Comparison between experiment and control groups according to the measuring axes at post measurement

\begin{tabular}{|c|c|c|c|c|c|c|}
\hline \multirow[t]{2}{*}{ The measuring axes } & \multicolumn{2}{|c|}{$\begin{array}{c}\text { Experiment } \\
(\mathrm{n}=10)\end{array}$} & \multicolumn{2}{|c|}{$\begin{array}{l}\text { Control } \\
(n=10)\end{array}$} & \multirow[t]{2}{*}{$\mathbf{t}$} & \multirow[t]{2}{*}{$\mathbf{P}$} \\
\hline & Mean & \pm ISD & Mean & \pm \pm SD & & \\
\hline Attention & 30.50 & 1.58 & 40.40 & 1.07 & $16.374^{*}$ & $\angle 0.001^{*}$ \\
\hline Impulsiveness & 24.50 & 1.90 & 31.40 & 1.07 & $9.994^{*}$ & $\angle 0.001^{*}$ \\
\hline Hyper activity & 22.20 & 2.25 & 25.50 & 0.85 & $4.337^{*}$ & 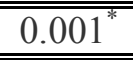 \\
\hline Overall & 77.20 & 3.88 & 97.30 & 1.25 & $15.585^{*}$ & $\overline{<<0.001^{*}}$ \\
\hline
\end{tabular}

t: Student t-test

$\mathrm{p}$ : $\mathrm{p}$ value for comparing between the studied groups

*: Statistically significant at $\mathrm{p} \leq 0.05$

Table No. 2 shows:

- A difference between the mean values of the experimental group and the mean values of the control group in terms of attention deficiency aspect favoring the experimental group as the values were; 40.40 and 30.50 respectively and the $t$ value was 16.394 , thus referring to significant differences at $\mathrm{P}<0.05(<0.001)$.

- Also there are differences between the mean values of the experimental group and the mean values of the control group on the impulsion aspect of the disorder favoring the experimental group, as the values were 25.50 and 24.50 respectively and $t$ value was 9.994, thus referring to significant differences at p $0.05(<0.001)$.

- There were differences in the mean values of the experimental group and the control group in terms of the hyperactivity aspect of the disorder favoring the experimental group and the values were 25.50 and 22.20 respectively while $t$ was 4.337 at $\mathrm{p} 0.05(<0.001)$.

The previous results show that there are significant differences between the mean values of the experimental group and the control group in terms of attention deficiency disorder scale as the mean values for the experimental group were 77.20 and for the control group 97.30 and $t$ value was 15.52, thus referring to significant differences at $\mathrm{P}<0.05(<0.001)$. 
Table (3): Comparison between pre and post measurement according to the measuring axes at experiment group

\begin{tabular}{|c|c|c|c|c|c|c|c|}
\hline \multirow{2}{*}{$\begin{array}{c}\text { The } \\
\text { measuring } \\
\text { axes }\end{array}$} & \multicolumn{2}{|c|}{$\begin{array}{c}\text { Pre } \\
(\mathrm{n}=10)\end{array}$} & \multicolumn{2}{|c|}{$\begin{array}{c}\text { Post } \\
(\mathrm{n}=10)\end{array}$} & \multirow{2}{*}{$\begin{array}{c}\% \\
\text { improvemen } \\
t\end{array}$} & \multirow{2}{*}{ T } & \multirow{2}{*}{$\mathbf{P}$} \\
\hline & $\begin{array}{c}\text { Mea } \\
\text { n }\end{array}$ & $\pm \mathrm{SD}$ & $\begin{array}{c}\text { Mea } \\
\text { n }\end{array}$ & \pm SD & & & \\
\hline Attention & 40.80 & 0.92 & 30.50 & 1.58 & $25.2 \%$ & 16.263 & $<0.001$ \\
\hline $\begin{array}{l}\text { Impulsivenes } \\
\text { s }\end{array}$ & 31.80 & 0.92 & 24.50 & 1.90 & $23.0 \%$ & 11.220 & $<0.001$ \\
\hline $\begin{array}{l}\text { Hyper } \\
\text { activity }\end{array}$ & 26.3 & 0.82 & 22.20 & 2.25 & $15.6 \%$ & $5.562^{*}$ & $<0.001$ \\
\hline Overall & 98.90 & 1.45 & 77.20 & 3.88 & $21.9 \%$ & 18.480 & $<0.001$ \\
\hline
\end{tabular}

t: paired t-test p: $p$ value for comparing between pre and post measurement

$*$ : Statistically significant at $\mathrm{p} \leq 0.05$

Table No. 3 shows:

- A difference between the mean values of the experimental group and the control group in both pre and post measurements in terms of the sub- scale "attention deficiency", as the mean value of the pre measurement is 40.80, while the mean value of the post measurement is 30.50 and $t$ value is 16.263 and the improvement percentage was $25.21 \%$, thus referring to significant differences at $\mathrm{p}<0.05(\mathrm{P}<0.001)$.

- A difference between the mean values of the experimental group in terms of pre and post measurements on the sub- scale of impulsion, for the mean value of the post measurement was 24.50 while the mean value of the pre measurement was 31.80 and $t$ value was 11.220 and the improvement percentage was $23 \%$, thus referring to significant differences at $\mathrm{P}<0.05(<0.001)$.

- The aforementioned shows a difference between mean values of experimental group's pre and post measurements on the attention deficiency scale, as the mean value of the pre measurement was 98.90, and the mean value of the post- measurement was 77.20 and $t$ value was 18.480 and the improvement percentage was $21.9 \%$ thus referring to the presence of significant differences at $p<0.05(<0.001)$. 
Table (4):Comparison between pre and post measurement according to the measuring axes at control group

\begin{tabular}{||l||c||c||c||c||c||c||}
\hline \multirow{2}{*}{ The measuring axes } & \multicolumn{2}{|c|}{ Pre(n= 10) } & \multicolumn{2}{|c||}{ Post(n= 10) } & \multirow{2}{*}{ t } & \multirow{2}{*}{ p } \\
\cline { 2 - 5 } & Mean & \pm SD & Mean & \pm SD & & \\
\hline \hline Attention & 40.40 & 0.70 & 40.40 & 1.07 & 0.000 & 1.000 \\
\hline \hline Impulsiveness & 31.50 & 1.27 & 31.40 & 1.07 & 0.190 & 0.853 \\
\hline \hline Hyper activity & 25.90 & 0.99 & 25.50 & 0.85 & 1.309 & 0.223 \\
\hline \hline Overall & 97.80 & 1.32 & 97.30 & 1.25 & 0.808 & 0.440 \\
\hline \hline
\end{tabular}

t: paired t-test $\mathrm{p}$ : $\mathrm{p}$ value for comparing between pre and post measurement

Table No. 4 showed that there is; no significant difference between the mean values of the control group and the pre and post measurements of the attention disorder scale and its sub- scales; attention deficiency, impulsion and hyperactivity, as $\mathrm{p}$ value was 0.440 and $\mathrm{t}$ value was 0.808 ; thus referring to the presence of significant differences between the two measurements.

\section{Discussion:}

The study proved the validity of the first hypothesis thereof, that there are differences in mean values of the experimental group and the control group in terms of the attention disorder scale favoring the experimental group, thus referring to the effectiveness of the intervention program using Augment Reality (AR) technique in reducing the attention deficiency disorder among children with learning disabilities favoring the post measurement of the experimental group. Therefore it confirms the effectiveness of using computers and 3D glasses in teaching different school subjects to the children with learning disabilities, which accords with the study of ElSaigh and Elsiddig (2017) which emphasized the importance of elearning in improving reading skills among the children with learning disabilities. This study also accords with the study of Bikic et al. (2018) which emphasized the positive impacts of cognitive training using computers with children with ADHD

Also the study proved the validity of its second hypothesis which refers to the presence of differences in the mean values of the experimental group in both the pre and post measurements in terms of the attention disorder scale favoring the post measurement as the improvement percentage was $21.5 \%$, which was the result of applying the intervention program. Suggesting the effectiveness of the Augmented Reality technique in reducing the attention disorder among children with learning disabilities. Using computers and 3D 
images positively affected the children's level of attention as the children's teachers have confirmed that there was a difference in children's level and period of attention, as well as, their ability to follow up lessons, increase in interest in activities and tasks and participation and finally a decrease in forgetting their lessons The study also mentioned that the improvement percentage in attention deficiency was $25.2 \%$, thus referring to the effectiveness of Augmented Reality techniques in reducing attention deficiency among children with learning disabilities.

The study showed that the improvement in impulsion was $23.0 \%$ as the children's teacher referred to the changes in children's level of impulsiveness manifesting in their commitment with the instructions given to them and reduction in their obvious impulsiveness in speech and reacting to others.

It also showed that the improvement percentage in hyperactivity was $15.6 \%$ As the children's teachers referred to the reduction in children's constant movement and their attraction to computers and 3D glasses; thus leading to limiting noise and chaos made by the ADHD children.

This indicates greater effect of the intervention program deploying Augmented Reality techniques in terms of degree of attention deficiency than in impulsion and that the least effect was in hyperactivity among children with learning disabilities.

The researcher considers this a result of the presence of other factors that increase impulsiveness and hyperactivity among children; such factors might be genetic or physiological which hinder the effect of Augmented Reality alone. This result agrees with that of several studies such as Yilmaz et al. (2017), Roschelle et al. (2000), Whitebread (2012), Cohen (2013), O'malley \& Fraser (2004), Mraschall (2007) and Glenber et al. (2004) for each of these studies has emphasized the effectiveness of teaching using Augmented Reality in empowering children to know, along with, limiting attention deficiency by different stimuli surrounding them. It also useful in increasing children's spatial awareness and providing them with jolly useful learning experiences. The study also indicated the validity of the third hypothesis, that there are no significant differences between the mean values of the control group and the experimental group in both of the pre and the post measurements in terms of the attention disorder and its sub- scales; attention deficiency, impulsion and hyperactivity; thus also suggesting the effectiveness of 
intervention program using augmented reality in reducing the attention disorder among children with learning disabilities.

\section{Conclusions:}

According to the study result; the researcher concluded the necessity to utilize the Augmented Reality technique through;

- Holding extensive training courses, workshops and seminars for teachers, educational professionals and social workers in using Augmented Reality apps in education in order to improve their level of performance using such technique.

- Changing the boring school textbooks into active ones with videos and 3D images; interactive books.

- Using the 3D glasses in education to improve the thinking and communication skills among learners; thus increasing their enthusiasm and will to learn.

\section{Limitation:}

The researcher had some difficulty in this research, as it took her a long time to use the AR technology, as well as, the difficulty in helping teachers and social workers to design computer activities throughout the research period. Generally applying AR requires small learning groups to achieve best advantage thereof, in addition to, the availability of good financial and technical resources for the application.

\section{Suggestions for future researches:}

The impact of using AR applications on academic achievement for the children with learning disabilities in middle school. The impact of using AR applications on academic achievement for the children with learning disabilities in high school. The impact of using AR applications on developing social skills of the children with learning disabilities. 


\section{References:}

Al-Asheeri, H.A.Y. (2017). Effectiveness of Using Augmented Reality Strategy in Enhancing Learning English for Cycle One Students in the Elementary Stage in the Kingdom of Bahrain.US-China Foreign Language, 15(7), p.409-419.doi:10.17265/15398080/2017.07.001

Alper, S., Lea Ryndak, D. \&Schloss, C.N. (2000).Alternate Assessment of Students with disabilities in Inclusive Settings. Boston, MA: Allyn\& Bacon.

Anderson, E. \&Liarokapis, F. (2014). Using Augmented Reality as a Medium to Assist Teaching in Higher Education. Present at:Conference: Eurographics,P.89

Aro, T. \&Ahonen, T. (2011). Assessment of Learning disabilities: Cooperation between Teachers, Psychologists and Parents. (African ed), Finland: University of Turku. p. 81-85.

Auriemma, D. L. (2016).Parenting stress in parents of children with learning disabilities. Fordham University, Pro Quest Dissertations Publishing, 10145751.

Azuma, R. T. (1997). A survey of augmented reality. Presence-Teleoperators and Virtual Environments, 6(4), 355-385.

Bikic, A., Leckman, J.F., Christensen, T.Ø., Bilenberg, N. \&Dalsgaard, S. (2018). Attention and executive functions computer training for attention-deficit/hyperactivity disorder (ADHD): results from a randomized, controlled trial, European Child \& Adolescent Psychiatry,27(12), 1563-1574.https ://doi.org/10.1007/s0078 7018-1151-y.

Boyce, D. A. (2016).The effect of the video game Quizlet on the acquisition of science vocabulary for children with learning disabilities. (Master Thesis), NJ: Rowan University.

Burke, J. W. et. al (2010). Augmented reality games for upper-limb stroke rehabilitation. Games andVirtual Worlds for Serious Applications (VS-GAMES), Second International Conference on, IEEE.

Butler, F., Miller, S., Crehan, K., Babbitt, B. \& Pierce, T.(2003). Fraction instruction for students with mathematical disabilities: Comparing two teaching sequences. Learning disabilities Research \& Practice, 18(2), 99-111.

Chandrasekera, T. \& Yoon, S. Y. (2015). Adopting Augmented Reality in Design Differences, 61, pp. 11-20. Cited 1 time Journal of Architectonic, Spatial, and Environmental Design, Volume 9, Issue 1, March, p. 1- 14.

Chandrasekera, T. \& Yoon, S.-Y. (2014). The effect of virtual reality and augmented reality in the design process. Paper presented at the $17^{\text {th }}$ Biannual Conference of the Design Communication Association, Atlanta, Georgia. 
Chen, X., Ye, M., Chang, L., Chen, W., Zhou, R. (2018). Effect of Working Memory Updating Training on Retrieving Symptoms of Children With Learning disabilities Journal of Learning disabilities, 51 (5), pp. 507-519..

Crealock, C., \&Kronick, D. (1993).Children and Young People with Specific Learning disabilities. Canada: UNESCO.

Daly, B.P. Creed, T.Xanthopoulos, M., \&Brown, R.T. (2007).Psychosocial treatments for children with attention deficit/hyperactivity disorder. Neuropsychological Review, 17, 73-89.

Elsaigh, A.M., Elsiddig, H.M. (2017). The role of e-learning in improving the reading skills of children with learning disabilities in reading from the point of view of teachers of children with learning disabilities Joint International Conference on Information and Communication Technologies for Education and Training and International Conference on Computing in Arabic, ICCA-TICET, art. no. 8095305.

Etmeezi, J. (2010). Nuthomaltaaleemalelektroni w adwatuh [E-learning systems and tools]. Phillipsburg, NJ: Phillips Publishing.p.55.

Fiedorowicz, C., et al (2015). "Position Paper. To Revise or Not To Revise: The Official LDAC Definition of Learning disabilities Versus DSM-5 Criteria". Canada: Learning disabilities Association of Canada.

Friedman, L.M., Rapport, M.D., Calub, C.A. \&Eckrich, S.J. (2018). ADHD and Core Foundational Learning: Working Memory's Contribution to Reading Comprehension and Applied Math Problem-Solving Abilities, The ADHD Report, 26(7), 1-7. 7https://doi.org/10.1521/adhd.2018.26.7.

Glenberg, A. M., Gutierrez, T., Levin, J. R., Japuntich, S., \&Kaschak, M. P. (2004). Activity and imagined activity can enhance young children's reading comprehension. Journal of Educational Psychology, 96(3):424.

Gorges, J., Neumann, P., Wild, E., Stranghöner, D., Lütje-Klose, B. (2018).

Reciprocal effects between self-concept of ability and performance: A longitudinal study of children with learning disabilities in inclusive versus exclusive elementary education.

Learning and Individual Differences, 61, 11-20.

Hall, J. G. (2015). Effects of child-centered play therapy on social skills, academic achievement, and self-concept of children with learning disabilities: A single-case design,(Doctoral dissertation), The University of North Carolina at Charlotte.

Hayes, M. (1993). You don't outgrow it: living with learning disabilities. Novato, CA: Academic Therapy Press.

Horowitz, S.H. (2014). The State of Learning disabilities: Facts, Trends and Emerging Issues, New York: National Center for Learning disabilities., ( $3^{\text {rd }}$ ed),p 3-5. 
Juanes, J. A. et al (2014). Augmented reality techniques, using mobile devices, for learning human anatomy. ACM International Conference Proceeding Series, pp. 7-11.

Kemp, G., Smith, M. \& Segal, J. (2015). Learning disabilities and Disorders:

Types of Learning Disorders and Their Signs. Available From:https://www.helpguide. org/articles/autism-learningdisabilities/learning-disabilities-and-disorders.htm?pdf $=12930$

King, J., Joseph, S. \& Katrina, S. (2017). Reimagining the Role of Technology in Education: Technology Plan Update, US: National Education American Institutes.

Marshall, P. (2007). Do tangible interfaces enhance learning? In:

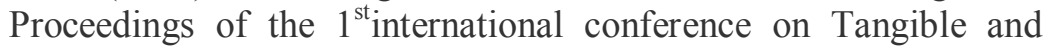
embedded interaction, pages 163-170. ACM

Martínez-Álvarez, P. (2017). Special ways of knowing in science: expansive learning opportunities with bilingual children with learning disabilities. Cultural Studies of Science Education, 12 (3), 521553.

Martín-Gutiérrez, J. et al (2010). Design and validation of an augmented book for spatial abilities development in engineering students. Computers \& Graphics, 34(1), 77-91.

Mason, E.J. \& Joshi, K.G. (2018).Non pharmacologic strategies for helping children with ADHD, Current Psychiatry, 17(1),P. 42-46.

McKeever, A.C.(2017). The Effects of Classical Music on Children with Learning disabilities in a Sixth Grade Mathematics Classroom, (Master Thesis), California: Rowan University.

Nidal, Abdel Ghafour.(2012). Educational frameworks for e-learning design. Al - Aqsa University Journal, 16(1), 63-86.

Olga S., Julie M.\&Cameron B. (2017). Augmented Reality Digital Technologies (ARDT) for Foreign Language Teaching and Learning, Principal Investigators: Funded SOTL Research: Indiana University's Communities of Inquiry.

Pierangelo, R. \& Giuliani, G. (2007). The educator's diagnostic manual of disabilities and disorders. San Francisco, CA: Jossey-Bass.

Qian, Z. (2018). The Application of Augmented Reality Visual Communication in Network Teaching.International Journal of Emerging Technologies in Learning, 13(7), 57-70.

Ribble, M. \& Bailey, G.D. (2015). Digital Citizenship in Schools International Society for Technology in Education ( $3^{\text {rd }}$ ed.). Eugene.

Roksolana, S. (2017). Augmented reality as a future of the education- Case study of Mole QL- educational augmented reality application. Master's thesis. Tallinn university of technology. 
Roschelle, J. M., Pea, R. D., Hoadley, C. M., Gordin, D. N. \& Means, B. M. (2000). Changing how and what children learn in school with computer-based technologies. Children and Computer Technology, 10(2), 76-101.

Seema, M. (2017). The Influence of Early Parent Involvement and Depression on Academic Achievement, Psychosocial Behaviors, and Motivation in Children with Learning disabilities Across Elementary School, (Doctoral Dissertations), Michigan State University. 10287920.

Tripp, G. \&Wickens, J.R. (2009).Neurobiology of ADHD. Neuropharmacology, 57, 579-589.

Tülü, B.K.\&Ergül, C. (2015). Perceiving nonverbal cues of affect in children with learning disabilities, Türk Psikoloji Dergisi, 30 (76), 32-47.

Valle, J.W. (2018). Across the Conference Table: Private and Public Mothering of Children With Learning disabilities. Learning Disability Quarterly, 41 (1), 7-18.

Whitebread, D. (2012). Developmental psychology and early childhood education: a guide for students and practitioners. UK: Sage Publishing.

Wood, E. \&Attfield, J. (2005). Play, learning and the early childhood curriculum. UK: Sage Publishing.

Yilmaz, R.M., Kucuk, S. \&Goktas, Y. (2017). Are augmented reality picture books magic or real for preschool children aged five to six? British Journal of Educational Technology, 48(3), 824-841. doi:10.1111/bjet.12452

Yonezawa.T.\&Tokuda, H. (2013). Second-sighted glasses: Discovering and understanding context-Aware services with augmented reality technique, Proceedings of the SICE Annual Conference, pp. 23862391.

Yuen, S. C.-Y., Yaoyuneyong, G., \& Johnson, E. (2011). Augmented reality: An overview and five directions for AR in education. Journal of Educational Technology Development and Exchange, 4(1),P. 119140. 